BRoCARD: 24 propositions de Fermat.

Delaporte: Sur la réforme du calendrier.

DU PAsquier: Sur les nombres transfinis.

Greenhill: Les fonctions de Fourier et Bessel comparées.

D'OCAGNE: La pratique courante de la méthode nomographique des points alignés: à propos de ses applications de guerre.

Grossmann: Sur l'état de publication des œuvres d'Euler.

Postiglione: Cyclómetrie mécanique.

Dubecq: Communication sur l'enseignement, République Argentine.

Zervos: Sur l'enseignement mathématique.

David Eugene Smith.

\title{
NOTE ON A METHOD OF PROOF IN THE THEORY OF FOURIER'S SERIES.
}

BY PROFESSOR DUNHAM JACKSON.

(Read before the American Mathematical Society September 7, 1920.)

IT has been pointed out on various occasions* that if $f(x)$ is a continuous function of period $2 \pi$ satisfying the LipschitzDini condition, that is, if $\lim _{\delta=0} \omega(\delta) \log \delta=0$, where $\omega(\delta)$ is the maximum of the oscillation of $f(x)$ in an interval of length $\delta$, then the uniform convergence of the Fourier series for $f(x)$ can be inferred almost immediately from the following two propositions:

$A . \dagger$ If $f(x)$ satisfies the Lipschitz-Dini condition, $\$$ there exists for every positive integral value of $n$ a finite trigonometric sum $\tau_{n}(x)$, of order $n$ at most, such that $\lim _{n=\infty} r_{n} \log n$ $=0$, where $r_{n}$ is the maximum of $\left|f(x)-\tau_{n}(x)\right|$.

* Cf., e.g., Lebesgue, "Sur les intégrales singulières," Annales de la Faculté de Toulouse, series 3, vol. 1 (1909), pp. 25-117; pp. 116-117.

$\dagger$ Cf., e.g., Lebesgue, loc. cit., p. 116; D. Jackson, "On the approximate representation of an indefinite integral, etc.," Transactions Amer. Math. Society, vol. 14 (1913), pp. 343-364; p. 350.

$\ddagger$ It is understood throughout the paper that every function considered has the period $2 \pi$. 
$B .^{*}$ If $\varphi(x)$ is any continuous $\dagger$ function, and $S_{n}(x)$ the partial sum of the Fourier series for $\varphi(x)$ to terms of order $n$, then $\left|S_{n}(x)\right|$ can not exceed $K M \log n$, where $M$ is the maximum of $|\varphi(x)|$, and $K$ is an absolute constant.

The central point in the proof is the fact that $\tau_{n}(x)$ is identical with the partial sum of its own Fourier series to terms of order $n$. It is the purpose of this note to show that similar reasoning can be applied to the arithmetical mean of Fejér, $\$$ in spite of the fact that the Fejér mean formed for a finite trigonometric sum $\tau_{n}(x)$ is not the same as $\tau_{n}(x)$. It is necessary to change the argument somewhat, but there is no difficulty in making the required modification.

Let $f(x)$ be now an arbitrary continuous function, and let $\sigma_{n}(x)$ be the arithmetical mean of the partial sums of the Fourier series for $f(x)$, to terms of order $n$. The uniform convergence of $\sigma_{n}(x)$ to the value $f(x)$ is to be deduced from the propositions:

$C$. (Weierstrass's theorem.) $\S$ If $f(x)$ is continuous, there exists for every positive integral value of $n$ a finite trigonometric sum $\tau_{n}(x)$, of order $n$ at most, such that $\lim _{n=\infty} r_{n}=0$, where $r_{n}$ is the maximum of $\left|f(x)-\tau_{n}(x)\right|$.

$D$. $\|$ If $\varphi(x)$ is any continuous function (more generally, any integrable function), and $\sigma_{n}(x)$ the Fejér mean of the Fourier series for $\varphi(x)$ to terms of order $n$, then $\left|\sigma_{n}(x)\right|$ can not exceed $M$, where $M$ is the maximum of $|\varphi(x)|$.

Let $\epsilon$ be any positive quantity. Let a finite trigonometric sum $\tau_{p}(x)$, of order $p$, be determined, according to Proposition $C$, so that

$$
\left|f(x)-\tau_{p}(x)\right|<\frac{1}{3} \epsilon .
$$

Let $\sigma_{n 1}(x)$ be the Fejér mean, of order $n$, for the function $\tau_{p}(x)$, and $\sigma_{n 2}(x)$ the corresponding mean for the function $f(x)-\tau_{p}(x)$.

* Cf., e.g., Lebesgue, loc. cit., p. 116; D. Jackson, "On approximation by trigonometric sums and polynomials," Transactions Amer. Math. Society, vol. 13 (1912), pp. 491-515; pp. 502, 512-515.

+ It is sufficient for the truth of the statement that $\varphi$ be integrable, but for present purposes there is no need of speaking of any but continuous functions.

‡Fejér, "Untersuchungen über Fouriersche Reihen," Mathematische Annalen, vol. 58 (1904), pp. 51-69.

\& Weierstrass, "Ueber die analytische Darstellbarkeit sogenannter willkürlicher Functionen einer reellen Veränderlichen," Berliner Sitzungsberichte, 1885, pp. 633-639, 789-805; p. 801.

\| Fejér, loc. cit., p. 60 . 
Then

$$
\sigma_{n}(x)=\sigma_{n 1}(x)+\sigma_{n 2}(x) .
$$

By Proposition $D$,

$$
\begin{gathered}
\left|\sigma_{n 2}(x)\right|<\frac{1}{3} \epsilon, \\
\left|f(x)-\tau_{p}(x)-\sigma_{n 2}(x)\right|<\frac{2}{3} \epsilon,
\end{gathered}
$$

for all values of $n$. The quantity $\sigma_{n 1}(x)$ is the arithmetical mean of $n+1$ finite trigonometric sums, of which all from the $(p+1)$ th on, if $n \geqq p$, are identical with $\tau_{p}(x)$, while each of the first $p$ is composed of a part of the terms of $\tau_{p}(x)$. Added together, the first $p$ sums which enter into the mean give a finite trigonometric sum $\omega_{p-1}(x)$, which is of order $p-1$ at most, and independent of $n$. So $\sigma_{n 1}(x)$ can be written in the form

$$
\begin{aligned}
\sigma_{n 1}(x)=\frac{\omega_{p-1}(x)+(n+1-p)}{n+1} & \\
& =\tau_{p}(x)+\frac{\omega_{p-1}(x)-p \tau_{p}(x)}{n+1} .
\end{aligned}
$$

As the last numerator is independent of $n, \sigma_{n 1}(x)$ approaches $\tau_{p}(x)$ uniformly as $n$ becomes infinite-a fact which is fairly obvious in the first place-and, if $n$ is sufficiently large,

$$
\left|\tau_{p}(x)-\sigma_{n 1}(x)\right|<\frac{1}{3} \epsilon .
$$

By combination of (1), (2), and (3), for values of $n$ satisfying (3),

$$
\left|f(x)-\sigma_{n}(x)\right|<\epsilon,
$$

which completes the convergence proof.

The University of Minnesota, Minneapolis, MinN. 